\title{
AVALIAÇÕES ACÚSTICA E PERCEPTIVA DE FALA NOS PROCESSOS DE DESSONORIZAÇÃO DE OBSTRUINTES
}

\author{
Perceptual and acoustical speech analyses on articulatory \\ disordered children with desonorization
}

\author{
Ana Paula Ramos de Souza ${ }^{(1)}$, Lisiane Collares Scott ${ }^{(2)}$, Carolina Lisbôa Mezzomo ${ }^{(3)}$, \\ Roberta Freitas Dias ${ }^{(4)}$, Vanessa Giacchini ${ }^{(5)}$
}

\begin{abstract}
RESUMO
Objetivo: comparar a percepção e a produção do traço de sonoridade de dois sujeitos, um em aquisição normal e outro com transtorno fonológico, e analisar as metodologias acústica e perceptual de investigação do traço [ \pm sonoro]. Procedimentos: um instrumento contendo pares mínimos com oposição do valor do traço sonoro foi criado para eliciar a fala dos sujeitos e proporcionar a análise acústica e perceptual do vozeamento em suas falas. Os dados de fala foram gravados em MiniDisc Sony MZ-R70 em sala tratada acusticamente e submetidos ao programa Sona-Graph 5500 da Kay Elemetrics, verificando a presença ou não de vozeamento. Dois clínicos e pesquisadores experientes em aquisição fonológica fizeram os julgamentos acerca do contraste de sonoridade na fala das crianças. Também se testou a discriminação auditiva do traço de sonoridade pelas crianças através do instrumento de Levi (1994). Resultados: ambas as crianças demonstraram perceber o traço de sonoridade e produzi-lo em alguns contextos. A criança em aquisição normal apresentou mais contextos com produção adequada do traço de sonoridade. Houve cerca de $10 \%$ de desacordo no julgamento do contraste de sonoridade entre os juízes. Conclusão: as análises acústicas e perceptuais são complementares na avaliação da fala. Existem momentos de dessonorização na fala do sujeito em aquisição típica.
\end{abstract}

DESCRITORES: Distúrbios da Fala; Acústica da Fala; Percepção da Fala; Reabilitação dos Transtornos da Fala e da Linguagem

(1) Fonoaudióloga; Professora Adjunta do Curso de Fonoaudiologia e do Programa de Pós-Graduação em Distúrbios da Comunicação Humana da Universidade Federal de Santa Maria, UFSM, Santa Maria, RS; Doutora em Linguística Aplicada pela Pontifícia Universidade Católica do Rio Grande do Sul.

(2) Fonoaudióloga; Fonoaudióloga na Clínica Clinifono; Mestre em Distúrbios da Comunicação pela Universidade Tuiuti do Paraná.

(3) Fonoaudióloga; Professora Adjunta do Curso de Fonoaudiologia e do Programa de Pós-Graduação em Distúrbios da Comunicação Humana da Universidade Federal de Santa Maria, UFSM, Santa Maria, RS; Doutora em Linguística Aplicada pela Pontifícia Universidade Católica do Rio Grande do Sul.

(4) Fonoaudióloga; Clínica Prefeitura Municipal de Vitória das Missões-RS; Mestre em Distúrbios da Comunicação Humana pela Universidade Federal de Santa Maria.

(5) Fonoaudióloga; Fonoaudióloga da Associação de Pais e Amigos dos Excepcionais de Marau-RS; Mestre em Distúrbios da Comunicação Humana da Universidade Federal de Santa Maria.

Conflito de interesses: inexistente

\section{INTRODUÇÃO}

Os estudos sobre o processo de aquisição fonológica normal e desviante vêm sendo de grande interesse em várias áreas do conhecimento, sobretudo na Fonoaudiologia. Nesse sentido, a literatura aponta a dessonorização como sendo um dos desvios mais frequentes e de maior dificuldade evolutiva na prática clínica. Por isso, a investigação das causas da dessonorização é de valor imensurável à prática clínica ${ }^{1-3}$.

Estudos fonológicos e fonéticos sobre a dessonorização referem a idade dos 5 anos como o momento de supressão do processo na aquisição normal do Português Brasileiro, podendo estar completamente superado antes disso ${ }^{4,5}$.

A estratégia de dessonorização representa a dificuldade na coordenação dos eventos glóticos e supraglóticos ${ }^{6,7}$. Um atraso no início da 
sonorização faz as oclusivas soarem como surdas, demonstrando um comprometimento na organização têmporo-espacial dos movimentos dos Órgãos Fonoarticulatórios (OFA) ${ }^{8}$, podendo ser afetado por treinamento e tipo de tarefa ${ }^{9}$, bem como por aspectos contextuais verbais ${ }^{10,11} \mathrm{e}$ não-verbais ${ }^{12} \mathrm{e}$ até dialetais ${ }^{13}$.

Outro aspecto que se deve investigar na análise da dessonorização é a percepção e sua relação com a produção. Questiona-se se as representações subjacentes da criança diferem das representações superficiais e se as alterações fonológicas originam-se em falhas na percepção, no armazenamento ou na produção motora.

Assim, este estudo pretendeu investigar o processo de dessonorização de obstruintes na fala de crianças com desenvolvimento fonológico normal e desviante, utilizando as análises perceptual e acústica. Além disso, buscou-se confrontar a capacidade perceptual e produtiva dos sujeitos para o traço de sonoridade.

\section{APRESENTAÇÃO DOS CASOS}

Participaram deste estudo dois sujeitos, um com distúrbio fonológico - S1, apresentando processo de dessonorização em suas produções e o outro com desenvolvimento normal de fala (DNF) - S2, ambos na faixa etária de 5 anos. A criança com fala normal participou da pesquisa como controle, a fim de se estabelecer parâmetros de comparação. A seleção dos sujeitos foi realizada na clínica escola da Universidade Luterana do Brasil - RS (ULBRA$R S$ ), e foi uma amostra por conveniência, já que foi selecionado um sujeito portador de transtorno fonológico e que ainda não havia iniciado atendimento fonoaudiológico e um sujeito controle de mesma idade em aquisição típica de linguagem, sobretudo no aspecto fonológico.

Para a seleção dos sujeitos, foram utilizados como critérios de inclusão a assinatura pelos responsáveis do Termo de Consentimento Livre e Esclarecido para autorização da pesquisa, o que Ihes garantiu direito de sigilo e voluntariado; um dos sujeitos deveria apresentar distúrbio fonológico e o outro com DNF, diagnosticados através da aplicação da Avaliação Fonológica da Criança - AFC ${ }^{14}$ e conversa espontânea; ambos os sujeitos deveriam apresentar idades aproximadas, superiores a 4:11 (idade de domínio do sistema fonológico do português brasileiro); não deveriam ter recebido atendimento fonoaudiológico prévio; não poderiam apresentar alterações de voz e/ou de motricidade orofacial, que pudessem interferir nas produções orais. O AFC é um instrumento que tem como objetivo eliciar uma amostra representativa da fala da criança através de nomeação e fala espontânea. É composto por cinco desenhos temáticos para a estimulação de 125 itens que formam a lista de palavras. Possibilita a ocorrência de sons em posições diferentes nas sílabas e na palavra.

Foram considerados critérios de exclusão para o sujeito com distúrbio fonológico a presença de alterações nos órgãos fonoarticulatórios; alterações no desenvolvimento da linguagem, exceto no nível fonológico; alteração nos limiares auditivos; alterações psicológicas, cognitivas e/ou neurológicas. O sujeito com DNF deveria apresentar desenvolvimento condizente com o esperado para a sua idade cronológica.

Foram investigadas nesta pesquisa, tanto a produção quanto e percepção do contraste de sonoridade dos dois sujeitos, com DNF e com distúrbio fonológico. Para a análise da produção realizou-se a análise acústica e a perceptiva auditiva através de dois julgadores em cada caso. A análise da percepção dos sujeitos para o contraste de [ \pm sonoro] foi avaliada por meio da oferta de pares mínimos, conforme será descrito a seguir.

Para coletar dados de fala das crianças e realizar as análises acústica e perceptivo auditiva por julgadores adultos, após a seleção dos sujeitos foi elaborado e aplicado um instrumento. Esse instrumento continha pares mínimos com contraste de sonoridade e diferentes pontos de articulação ([p/b, f/v, k/g, S/Z]) em onset inicial e medial, com três ofertas por posição em cada par, totalizando 24 itens no teste. Cada palavra foi representada por uma figura e apresentada em pranchas diferentes às crianças. As mesmas deveriam produzir a palavra que representava o desenho, apresentado pelo avaliador na primeira e única tentativa. Todos os acertos e erros foram computados e analisados comparativamente entre os sujeitos, tanto perceptual quanto acusticamente.

A fim de realizar a avaliação acústica, foram utilizadas as amostras obtidas por meio da aplicação do instrumento anteriormente referido, composto pelos pares mínimos, utilizados na coleta de dados de fala. Estas amostras foram gravadas através da utilização de um MiniDisc Sony MZ-R70 e microfone LeSon MP68, padrão polar cardioide, eletreto, de baixa impedância, com chave e cabo balanceado. As gravações foram realizadas no laboratório de voz e fala da clínica escola (tratado acusticamente) e analisadas por meio do programa Sona-Graph 5500 da Kay Elemetrics DSP. A taxa de amostragem utilizada foi de $25000 \mathrm{~Hz}$ e o volume, de três pontos do aparelho.

Por meio da espectrografia foram analisadas a presença ou ausência do vozeamento por duas julgadoras, uma professora e outra estudante do 
último ano da graduação do curso de fonoaudiologia da ULBRA-RS. Considerou-se que, como na língua portuguesa as plosivas são caracterizadas por VOT negativa, a presença de barra de sonoridade precedendo o burst já se constitui em indício de que a criança estaria produzindo plosivas vozeadas, optou-se pela análise da presença da mesma para decidir pela presença ou ausência de sonoridade.

Para a avaliação perceptivo auditiva das produções dos sujeitos, as amostras analisadas acusticamente foram ouvidas e transcritas por dois julgadores fonoaudiólogos, com experiência na área. Esse procedimento foi realizado em momentos diferentes, sem contato entre os profissionais. Os julgadores não souberam dos achados da análise acústica, até que todas as análises perceptivas fossem completadas.

Por último, para a avaliação da discriminação do traço sonoro pelos sujeitos, utilizou-se o teste perceptivo de pares mínimos sonoro-surdo de ${ }^{15}$. Este teste é composto por pranchas com desenhos de pares mínimos. Uma das pesquisadoras solicitava (falava o nome de uma das figuras) e a criança deveria apontar para a correta. As respostas corretas e incorretas foram computadas na primeira tentativa, em uma tabela criada pelas pesquisadoras.

Este projeto foi aprovado por Comissão de Aconselhamento em Ética em Pesquisa do Curso de Fonoaudiologia da ULBRA, com o protocolo de aprovação n 107/2002.

Os resultados obtidos foram analisados de forma qualitativa.

\section{RESULTADOS}

Na comparação dos resultados entre o S1 e S2, fica clara a diferença de sonoridades na produção das plosivas e fricativas, com superioridade nos percentuais de sonoridade das fricativas em relação às plosivas. Como era esperado, em todas as análises de fala, S2 apresentou resultados de sonoridade abaixo do S1 (Figura 1).

Quanto à presença de sonoridade na produção de plosivas pelo S2, a análise acústica demonstrou ausência de sonoridade, enquanto que os examinadores I e II verificaram auditivamente a presença do traço. Os percentuais dos examinadores, no entanto, divergem em quase $10 \%$, o que ressalta a importância da análise acústica na precisão do diagnóstico de dessonorização (Figura 1).

Com relação às fricativas foi verificada presença de sonoridade em todas as análises realizadas. Dentre as análises, a acústica foi aquela que apresentou os maiores percentuais de sonoridade, valores próximos às porcentagens encontradas pelo examinador I. Os valores percentuais obtidos pela análise do examinador são de, aproximadamente, a metade das demais análises (Figura 1).

Com relação à percepção/discriminação do traço de sonoridade pelos sujeitos (Figura 2), percebe-se que S1 não apresenta diferenças nem dificuldade na percepção do traço de sonoridade em plosivas e fricativas. Já os resultados de S2 demonstram uma dificuldade na percepção do traço "sonoro" nas fricativas.

Os resultados de $\mathrm{S} 2$ demonstram uma dificuldade na percepção do traço "sonoro" nas fricativas.

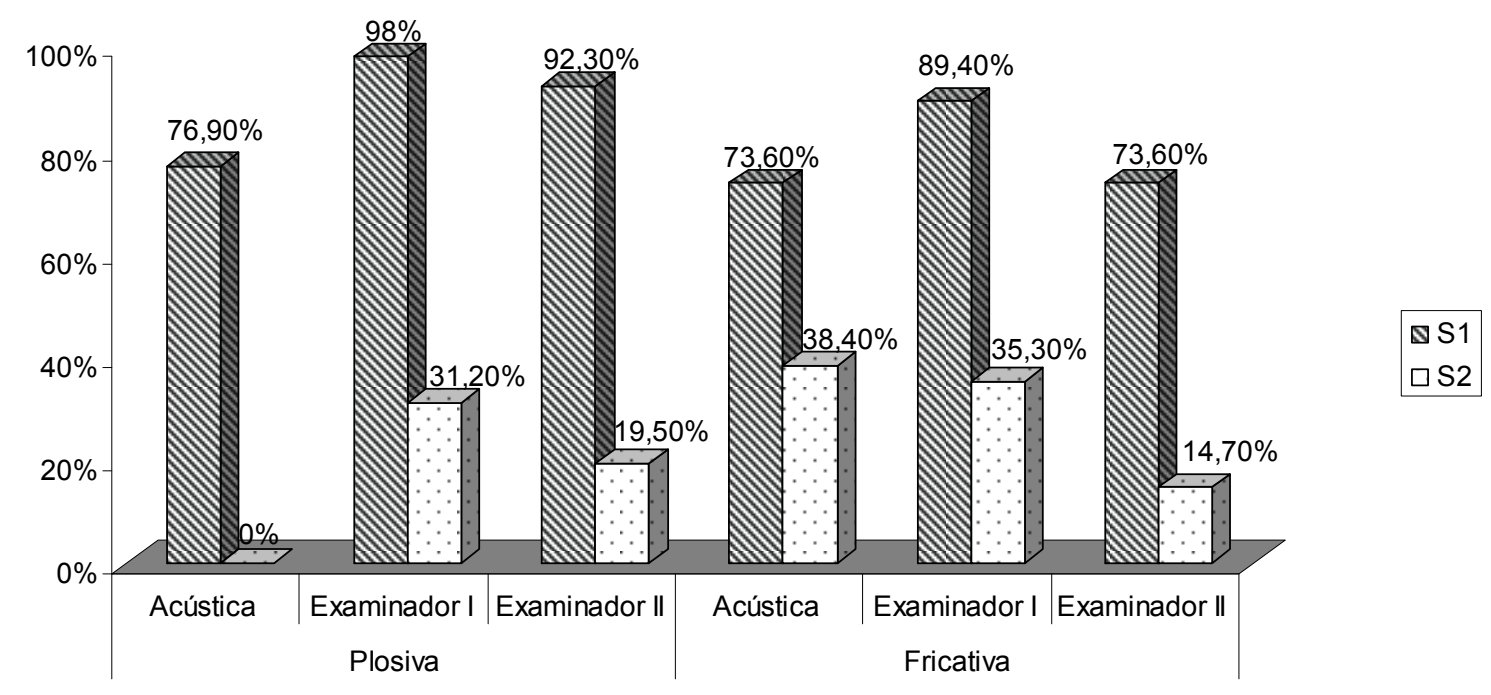

Figura 1 - Comparação entre a presença do traço de sonoridade em plosiva e fricativas, nas diferentes análises realizadas no sujeito controle (S1) e no sujeito pesquisado (S2) 


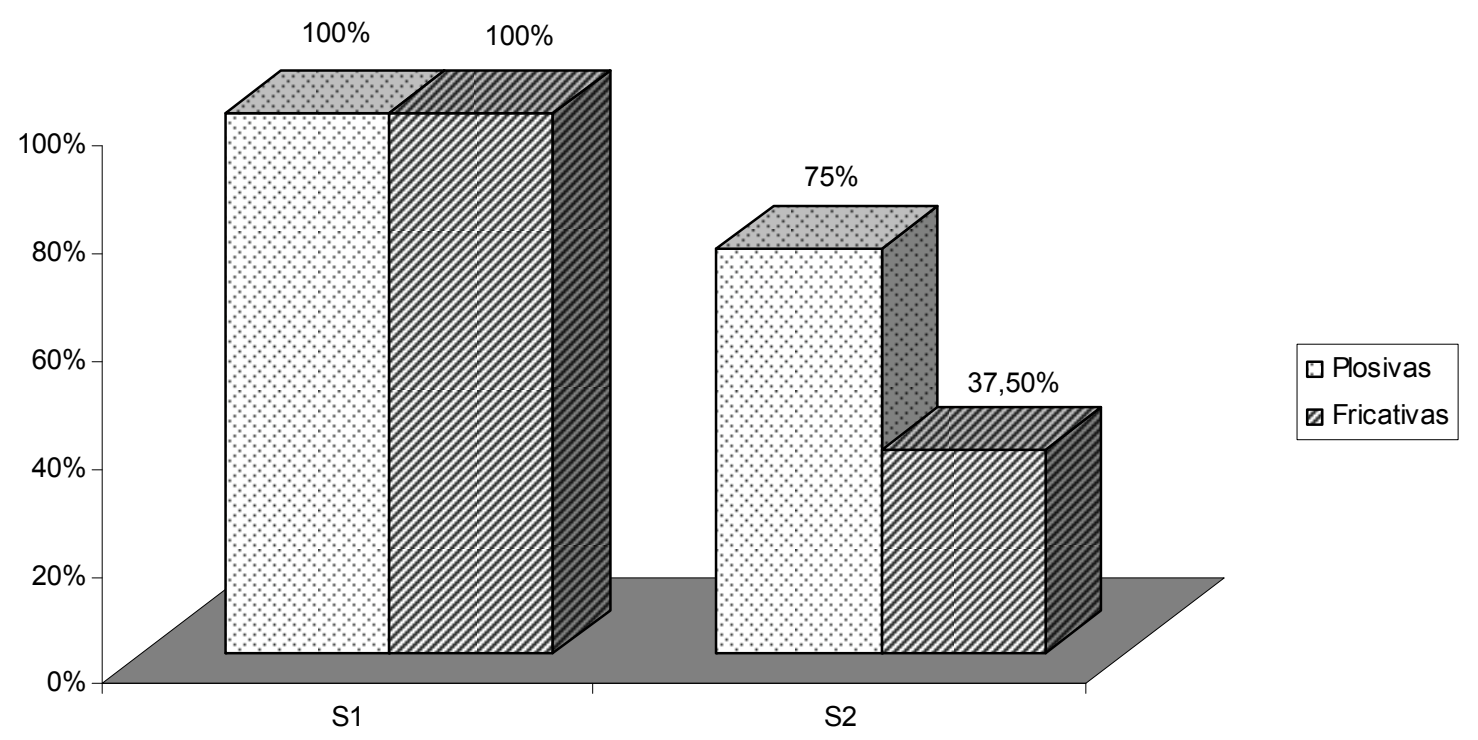

Figura 2 - Comparação entre a percepção do traço de sonoridade em plosivas e fricativas pelo sujeito controle (S1) e sujeito pesquisado (S2)

Ao contrastar os resultados das Figuras 1 e 2, verifica-se que S2 apresenta valores percentuais muito melhores na identificação do que na produção da sonoridade das plosivas. Observa-se uma divergência entre percepção e produção de sonoridade em relação às plosivas e fricativas (Figura 2).

\section{DISCUSSÃO}

Os resultados evidenciam uma porcentagem maior de ocorrências de dessonorização para as plosivas de S1 e S2 e fricativas de S1 por meio da espectrografia, quando comparados com a análise perceptivo auditiva. Tais resultados concordam com a análise acústica realizada, pela comparação da produção do traço de sonoridade para as plosivas /b/, /d/ e /g/ entre um grupo de crianças disfônicas, com maior ocorrência de dessonorização, e um grupo de crianças sem alterações de fala ${ }^{2}$. Esse tipo de estudo, demonstra que, por meio de análises fonético-acústicas e interpretação dos espectrogramas, relações entre os aspectos físicos e linguísticos podem ser identificadas ${ }^{6,11,13}$, auxiliando na compreensão da correlação entre o processo articulatório e o evento acústico resultante, e da relação entre os processos de produção e percepção da fala ${ }^{10,12}$. Este tipo de análise permite relacionar os distúrbios da fala, linguagem, voz e audição com os processos fisiológicos envolvidos em sua ocorrência ${ }^{16}$, bem como demonstrar que a criança tenha conhecimentos fonológicos não visualizáveis em sua produção motora ${ }^{17-19}$.

Ao contrastar os resultados apresentados na Figura $1 \mathrm{com}$ os resultados da Figura 2, nota-se que apesar de a percepção de S1 atingir 100\%, a sua produção para o traço sonoro apresenta-se mais rebaixada tanto para as plosivas como para as fricativas. Outros autores também observaram a antecedência da percepção sobre a produção ${ }^{5,19} \mathrm{e}$ o constante ajuste da percepção para monitorar pistas acústicas, seja em sujeitos em aquisição normal e com desvio ${ }^{20}$.

Para o S2 verifica-se que o valor percentual é melhor na percepção comparado aos valores percentuais de produção da sonoridade das plosivas. A análise acústica demonstrou uma proximidade entre a produção e a percepção das fricativas para este sujeito. Por outro lado, a percepção do traço sonoro das fricativas diverge da produção quando considerado os examinadores I e II para o S2 (Figura 2).

Esses resultados reforçam a idéia de que a percepção é determinada por um complexo de sinais acústicos. Como já foi evidenciada em outros trabalhos, tal complexidade pode ser responsável por mudanças na percepção de certas peculiaridades acústicas de um fonema ${ }^{16}$.

Sabe-se que as possibilidades perceptuais infantis já estão relativamente semelhantes a adultos entre 5 e 7 anos e que distinções entre faixas etárias podem estar mais relacionadas à experiência com a tarefa do que a possibilidades fisiológicas da criança ${ }^{18}$. Há estudos que demonstram capacidade importante de distinguir pelo Voice Onset Time (VOT) o traço de sonoridade entre 8,5 e 12 meses, com evolução entre ambas as idades no manejo de pistas acústicas ${ }^{19}$, e outros que demonstram que há múltiplas pistas acústicas atuando na identificação do contraste de sonoridade, que afeta tanto sujeitos 
em aquisição típica quanto atípica ${ }^{20}$. Entre todas as medidas acústicas, vários estudos demonstram que o VOT é uma medida muito robusta na análise de sonoridade ${ }^{21,22}$.

As crianças com distúrbio fonológico podem ser incapazes de manipular todos os sinais acústicos de um fonema, ou por uma imaturidade fisiológica, ou porque todos os sinais não são igualmente percebidos por elas. Um exemplo disso são as distinções de vozeamento que podem ser percebidas por crianças com distúrbio fonológico não só por distinção temporal do intervalo do VOT, mas pela realização independente de sinais de vozeamento, como por exemplo, início do F1, frequência fundamental ou amplitude do burst. Isso sugere que a criança com distúrbio fonológico pode conhecer algumas diferenças entre os cognatos vozeados e desvozeados, mas é incapaz de produzir todos os gestos que sinalizam tal contraste ${ }^{17}$. Tal imaturidade é comum em crianças menores em fase de aquisição 5,18,19.

$\mathrm{Na}$ aquisição fonológica do português brasileiro considerada normal a aquisição da classe das plosivas antecede a das fricativas ${ }^{4}$. Com isso, acredita-se que para o S2 a percepção acompanhe esta ordem de aquisição e sua produção, melhor para as fricativas, seja determinada por fatores motores.

Outro fato demonstrado neste gráfico é a melhor percepção do S2 para as plosivas do que fricativas, demonstrando resultados divergentes entre percepção e produção na análise da estrutura silábica, o que pode estar relacionado à alternância de acesso entre o léxico fonológico (com o traço de sonoridade presente) e o motor (com o traço de sonoridade ausente) ${ }^{23}$.
O estudo demonstra que a análise espectrográfica é um recurso que pode ser usado na verificação da forma como a criança está produzindo um determinado som, o que auxilia na adequação de sua produção, além de fornecer um parâmetro de comparação confiável dos dados de fala antes e após a terapia ${ }^{1-3}$.

\section{CONCLUSÃO}

Este estudo demonstra que há uma diferença na relação entre percepção e produção do traço de sonoridade. A criança com distúrbio fonológico percebe melhor uma classe de sons, mas produz melhor outra (melhor percepção nas plosivas e melhor produção nas fricativas).

O modo de articulação parece ser um parâmetro importante também na produção de sonoridade. As fricativas são mais produzidas do que as plosivas, verificadas através da análise acústica e perceptual para o sujeito com distúrbio fonológico.

A análise acústica mostrou-se fundamental para a avaliação da sonoridade, dado o alto índice de desacordo entre as análises perceptivo-auditivas dos examinadores entre si e delas com a análise acústica. Uma avaliação perceptiva imprecisa pode conduzir o terapeuta a opções não tão adequadas na prática clínica, podendo levar à demora no tratamento.

Há, assim, a necessidade de retomar e aprofundar os estudos nesta área ficando como sugestão a avaliação de um número maior de sujeitos com distúrbio fonológico e grupo controle.

\section{ABSTRACT}

Purpose: to compare perception and production of voiced constraint in the speech of two subjects, one in normal acquisition and another with phonological impairment, and analyzing acoustic and perceptual research methods of the voiced constraint. Procedures: an instrument of minimal voiced pair versus voiceless constraints were created to expel the speech production and perception of the subjects. The data acoustical analyses were recorded on MiniDisc Sony MZ-R70, in acoustic treated room, and by Sona-Graph 5500 program of Kay Elemetrics analysis. Two clinical and phonological acquisition researchers made perception judgments about speech of the children in terms of voiced and voiceless constraint. We also evaluated the voiced constraint perception by the children with Levi's instrument (Levi, 1994). Results: both children had appropriate perception of voiced constraint and its production in some contexts. The normal phonology acquisition had more contexts with adequate production. There were $10 \%$ of disagreement between the judges about children' voice constraint. Conclusion: the perception and acoustical analyses are complementary in speech evaluation. There are some voiceless events in the typical acquisition child's speech.

KEYWORDS: Speech Disorders; Speech Acoustics; Speech Perception; Rehabilitation of Speech and Language Disorders 


\section{REFERÊNCIAS}

1. Pagan LO, Wertzner HF. Intervenção no distúrbio fonológico por meio dos pares mínimos com oposições máximas. Pró-Fono. 2002; 14(3):313-24. 2. Arnaut MA, Ávila CRB. Ensurdecimento de fonemas plosivos na fala de crianças disfônicas. Rev. Soc. Bras. Fonoaudiol. 2008; 13(1):37-44.

3. Pagan LO, Wertzner HF. Análise acústica das consoantes líquidas do Português Brasileiro em crianças com e sem transtorno fonológico. Rev. Soc. Bras. Fonoaudiol. 2007; 12(2):106-13.

4. Fronza CA Uma tipologia alternativa para a aquisição do nó laríngeo e do nó de ponto de $\mathrm{C}$ por crianças falantes do PB. Signum Estud Linguag. 2004; 7(1):79-93.

5. Grigos Ml. Changes in articulator movement variability during phonemic development: a longitudinal study. J Speech Lang Hear Res. 2009; 52(1):164-77.

6. Zeroual C, Esling JH, Crevier-Buchman L. The contribution of supraglottic laryngeal adjustments to voice: phonetic evidence from Arabic. Logoped Phoniatr Vocol. 2008; 33(1):3-11.

7. Lowenstein JH, Nittrouer S. Patterns of acquisition of native voice onset time in English-learning children. J Acoust Soc Am. 2008; 124(2):1180-91.

8. Pincas J, Jackson PJ. Amplitude modulation of turbulence noise by voicing in fricatives. $\mathrm{J}$ Acoust Soc Am. 2006; 120(6):3966-77.

9. McCrea CR, Morris RJ. Effects of vocal training and phonatory task on voice onset time. J Voice. 2007; 21(1):5463.

10. Hanson HM. Effects of obstruent consonants on fundamental frequency at vowel onset in English. J Acoust Soc Am. 2009; 125(1):425-41.

11. Snoeren ND, Segui J, Hallé PA. Perceptual processing of partially and fully assimilated words in French. J Exp Psychol Hum Percept Perform. 2008; 34(1):193-204.

12. Elangovan S, Stuart A. Natural boundaries in gap detection are related to categorical perception of stop consonants. Ear Hear. 2008; 29(5):761-74.
13. Jacewicz E, Fox RA, Lyle $S$. Variation in stop consonant voicing in two regional varieties of American English. J Acoust Soc Am. 2008; 124(4):2559.

14. Yavas MS, Hernadorena CLM, Lamprecht RR. Avaliação fonológica da criança: reeducação e terapia. Porto Alegre: Artes Médicas; 2002.

15. Levy IP. Análise espectográfica do vozeamento de obstruintes em crianças de idade escolar. [tese]. Campinas: Universidade Estadual de Campinas; 1993.

16. Bharadwaj SV, Graves AG. Efficacy of the discreteness of voicing category (DOVC) measure for characterizing voicing errors in children with cochlear implants: a report. J Speech Lang Hear Res. 2008; 51(3):629-35.

17. Forrest K, Rockaman BK. Acoustic and perceptual analysis of word-initial stop consonants in phonologically disordered children. J Speech Hear Res. 1988; 31:449-59.

18. Nittrouer S, Lowenstein JH. Children's weighting strategies for word-final stop voicing are not explained by auditory sensitivities. J Speech Lang Hear Res. 2007; 50(1):58-73.

19. White KS, Peperkamp S, Kirk C, Morgan JL. Rapid acquisition of phonological alternations by infants. Cognition. 2008; 107(1):238-65.

20. Idemaru K, Holt L. Retuning speech sound categories: an eyetracking study. J Acoust Soc Am. 2008; 124(4):2438.

21. Francis AL; Kaganovich N; Driscoll-Huber C. Cue-specific effects of categorization training on the relative weighting of acoustic cues to consonant voicing in English. J Acoust Soc Am. 2008; 124(2):1234-51.

22. Theodore RM, Miller JL. Characteristics of listener sensitivity to talker-specific phonetic detail. J Acoust Soc Am. 2008; 124(4):2438.

23. Ramos APF Processos de Estrutura Silábica em Crianças com Desvios Fonológicos Evolutivos. Letras de Hoje. 1997; 32(4):49-59.

http://dx.doi.org/10.1590/S1516-18462010005000039

RECEBIDO EM: 21/10/2009

ACEITO EM: 24/01/2010

Endereço para correspondência:

Ana Paula Ramos de Souza

Rua Raposo Tavares 134 ap. 401

Santa Maria - RS

CEP: 97015-560

E-mail: ramos1964@uol.com.br 